\title{
A Foreword to "Classical Theory of Charged Point-Particles with Dipole Moments" by C. M. Lattes, M. Schönberg, and Walter Schützer
}

\author{
Alberto Saa ${ }^{1}$ \\ Accepted: 31 December 2021 / Published online: 31 January 2022 \\ (c) The Author(s) under exclusive licence to Sociedade Brasileira de Física 2022
}

The following paper [1], by C. M. Lattes, M. Schönberg, and W. Schützer, appeared in the September 1947 issue (volume 19, pages 193-245) of the Anais da Academia Brasileira de Ciências, the official scientific journal of the Brazilian Academy of Sciences. It was one of the first theoretical papers produced in the Department of Physics of the University of São Paulo (USP). The three authors were students at USP of Gleb Wataghin, a Russian-Italian physicist widely recognized as one of the founding fathers of Brazilian Physics. In particular, he was one of the founders of the Department of Physics at USP, in 1934. The paper, hence, is one of the first authentic Brazilian contributions to Physics literature. Curiously, C. M. Lattes, a graduate in Physics in the class of 1943 at USP, was an accomplished experimental physics, and this is one of his rare pure theoretical contributions. On the other hand, Schönberg (Mathematics, class of 1936) and Schützer (Physics, class of 1942) were both theoreticians with strong mathematical backgrounds.

The paper presents an extension of Dirac's classical model $[2,3]$ for the electron. Dirac's original proposal was plagued with many well-known difficulties, ranging from the so-called Abraham-Lorentz-Dirac radiation reaction force to some acausal solutions. In a previous work, Schönberg [4] proposes some strategies to deal with these difficulties involving, in the context of Maxwell's equations, the division of the total field created by a point charge in two parts, with only one of them accounting for the radiation emission. Lattes, Schönberg, and Schützer's contribution consists in the extension of the Schönberg proposal for the case of charged point particles with electric or magnetic dipole moments.

Although we know today that the solution of the radiation reaction force paradoxes must involve Quantum Mechanics and details of the causal structure of the spacetime (see, for instance, [5] and [6], respectively, and the references therein), such earlier approaches as [1], entirely formulated in the context of classical electrodynamics, are still interesting for a deeper understanding of these fundamental problems and the limitations of Classical Physics.

\section{References}

1. C.M. Lattes, M. Schönberg, W. Schützer, Classical theory of charged point-particles with dipole moments, Braz. J. Phys. (2022). https://doi.org/10.1007/s13538-021-01049-5

2. P.A.M. Dirac, The classical theory of electron, Proc. Royal Soc. A 167, 148 (1938)

3. P.A.M. Dirac, La théorie de l'électron et du champ électromagnétique, Ann. Inst. Poincaré 9, 13 (1938)

4. M. Schönberg, Classical theory of the point electron, Phys. Rev. 69, 211 (1947)

5. A. Higuchi, Radiation reaction in quantum field theory, Phys. Rev. D 66, 105004 (2002). [arXiv:quant-ph/0208017]

6. C. de Almeida, A. Saa, The radiation of a uniformly accelerated charge is beyond the horizon: a simple derivation, Am. J. Phys. 74, 154 (2006). [arXiv:physics/0506049]

Publisher's Note Springer Nature remains neutral with regard to jurisdictional claims in published maps and institutional affiliations.
Alberto Saa

asaa@ime.unicamp.br

1 Instituto de Matemática, Estatística e Computação Científica, Universidade Estadual de Campinas, Rua Sérgio Buarque de Holanda, 651, Campinas, São Paulo 13083-859, Brazil 